\title{
Diagnostic Utility of Pleural Fluid and Serum Markers in Differentiation between Malignant and Non-Malignant Pleural EFFUSIONS
}

\author{
P. Korczynski ${ }^{1}$, R. Krenke ${ }^{1}$, A. Safianowska ${ }^{1}$, K. Gorska ${ }^{1}$, B. M. Abou Chaz ${ }^{1}$, M. Maskey-Warzechowska ${ }^{1}$, \\ A. Kondracka ${ }^{2}$, J. Nasilowski ${ }^{1}$, R. Chazan ${ }^{1}$ \\ ${ }^{1}$ Department of Internal Medicine, Pneumology and Allergology and ${ }^{2}$ Department of Internal Medicine and Endocrinology, Medical \\ University of Warsaw, Poland
}

\begin{abstract}
Study objective: To evaluate the diagnostic value of four different tumor markers: cancer antigen 125 (CA125), carcinoembryonic antigen (CEA), cytokeratin 19 fragment (CYFRA 21-1) and neuron specific enolase (NSE) in patients with malignant and non-malignant pleural effusion.

Material and methods: One hundred and two patients with pleural effusion treated in the University Hospital in Warsaw between 2001 and 2003 were studied. They underwent an extensive, diagnostic work-up in order to determine the pleural effusion etiology. Patients with known pleural fluid etiology were labeled as the study group and submitted for further analysis. Pleural fluid and serum samples for CA-125, CEA, CYFRA 21-1 and NSE measurements were collected during the first thoracentesis, centrifuged, and frozen until further use. Pleural fluid and serum concentration of tumor markers were assessed by electrochemiluminescence methods using commercial kits.

Results: 74 patients ( $32 \mathrm{M}, 42 \mathrm{~F}$; mean age $65 \pm 14$ years) composed the final study group. Exudative pleural effusion was found in 62 patients; of these 36 were malignant (48.6\% of all effusions), 20 parapneumonic (or pleural empyema), and 6 tuberculous. In 12 patients, pleural transudate was diagnosed. The highest diagnostic sensitivity for malignant pleural effusion was found for NSE (94.4\% and 80.6\% in the pleural fluid and serum, respectively). However, the specificity of NSE measurement was relatively low $(36.1 \%$ and $47.4 \%$ in pleural fluid and serum, respectively). The most specific markers of malignant pleural fluid etiology were pleural fluid CYFRA 21-1 and CEA levels (92.1\% and $92.1 \%$, respectively). CA-125 was found to be the most specific serum marker of pleural malignancies $(78.9 \%)$. The AUC for combined pleural markers was 0.89, combined serum markers 0.82, combined ratio pleural/serum markers 0.88 .

Conclusions: There are significant differences between the diagnostic value of various pleural fluid and serum markers. Overall, pleural fluid markers are superior to serum markers in determining the pleural fluid etiology. A combination of two or more tumor markers may help improve their diagnostic accuracy. Pleural fluid and serum measurements of different tumor markers
\end{abstract}

play a limited role in the differentiation between malignant and non-malignant pleural effusions.

Key words: pleural effusion, cancer antigen-125, neuron specific enolase, carcinoembryonic antigen

\section{INTRODUCTION}

Pleural effusion (PE) may occur in many different diseases and is often a diagnostic challenge. One of the main issues in the differential diagnosis of PE is distinguishing exudates from transudates. This requires the assessment of the physicochemical properties of the fluid and comparison of selected biochemical parameters (e.g., total protein, lactate dehydrogenase activity, bilirubin, and cholesterol) in the fluid and serum. Determining the nature of PE (exudate or transudate) allows reducing the list of potential PE causes and indicates the direction for further diagnosis.

Another important clinical issue is the etiology of effusion - malignant or benign. This is crucial for PE management and prognosis. Cytological examination of PE samples is a simple and widely used diagnostic tool for differentiating the origin of the pleural fluid. It is characterized by a high specificity; however it has a relatively low sensitivity (40-87\%, mean ca 60\%) [1]. Confirming the malignant PE etiology in the patient with a negative cytological PE examination requires more complicated diagnostic procedures, such as needle pleural biopsy or thoracoscopy. Thus, in a number of patients the distinction between malignant and non-malignant PE may be difficult and limited by the risk related to invasive procedures. Tumor markers seem to be a promising alternative and have been proposed to aid in the differentiation of the PE etiology. These include carcinoembryonic antigen (CEA), cancer antigens: CA125, CA 15-3, CA 19-9, CA 72-4, cytokeratin fragments (CYFRA 21-1), neuron specific enolase (NSA), and squamous cell carcinoma antigen (SCCA). However, the clinical value of these markers is still unclear.

Several studies revealed that the level of some tumor markers in the pleural fluid and/ or serum is a reliable predictor of malignant PE etiology [2]. Other studies show that the sensitivity, specificity and diagnostic accuracy of different tumor markers are quite 
low [3]. Therefore, the aim of the present study was to evaluate the diagnostic value of four selected tumor markers: cancer antigen 125 (CA-125), carcinoembryonic antigens (CEA), cytokeratin 19 fragment (CYFRA 21-1), and neuron-specific enolase (NSE), measured in the pleural fluid and in serum in differentiation between malignant and non-malignant pleural effusions.

\section{Material AND Methods}

SuBJECTS

The study protocol was approved by the institutional Ethics Committee. One hundred and two pleural fluid and serum samples were prospectively collected from 102 patients with pleural effusion admitted to the Department of Internal Medicine, Pneumology and Allergology, Medical University of Warsaw, Poland, between 2001 and 2003. All patients were evaluated according to the well established diagnostic algorithm, including thoracentesis, closed pleural biopsy, and thoracoscopy [4]. Light's criteria were used to discriminate exudative and transudative pleural effusions. The effusion was considered malignant, if malignant cells or malignant tissue was found in the pleural fluid or pleural biopsy samples, respectively. Pleural effusions in patients with known malignant disease were also classified as malignant, unless alternative pleural fluid causes were diagnosed [5] At least one of the two criteria had to be met to recognize tuberculous pleurisy: 1) positive culture for $M$. tuberculosis in the pleural fluid, pleural biopsy, fibrinous adhesions collected during thoracoscopy or respiratory samples (sputum, bronchial washing, BALF), 2) caseating granulomas in pleural biopsy samples [6]. Parapneumonic effusion or pleural empyema were diagnosed in patients who had: 1) grossly purulent pleural effusion; 2) the presence of microorganisms in pleural fluid; 3) acute febrile illness, pleuritic chest pain, pulmonary infiltrates on chest radiograph and no alternative explanation for pleural fluid formation [7]. Congestive heart failure was recognized in patients with physical signs of heart failure (peripheral edema, crepitations on chest auscultation), cardiomegaly, and radiographic signs of pulmonary venous congestion. Seventy-four patients with known pleural fluid etiology were selected for further analysis. In the remaining 28 patients, the etiology of effusion was uncertain (these included 10 subjects who refused further evaluation) and those patients were not further evaluated.

Pleural fluid (mean volume $50 \mathrm{ml}$ ) and blood samples for tumor marker measurements were collected during the first thoracentesis. They were centrifuged at $2000 \mathrm{rpm}$ for $10 \mathrm{~min}$ and the supernatant was frozen at $-20^{\circ} \mathrm{C}$ until assayed. Concentrations of CEA, CA125, CYFRA 21-1, and NSE were measured using electrochemiluminescence immunoassays on Roche Elecsys 1010 analyzer (Roche Diagnostics; Mannheim, Germany).

\section{STATISTICAL ANALYSIS}

Statistical analysis was performed using statistical software package MedCalc 9.5.2.0. Data are presented as the median and interquartile range (IQR). The nonparametric Mann-Whitney U-test was used to test for significance between malignant and non-malignant PEs. Receiver operating characteristic (ROC) analysis was performed to quantify the accuracy of various markers to discriminate between malignant and benign PEs. The utility of each tumor marker was determined by means of sensitivity, specificity, positive predictive value (PPV), negative predictive value (NPV), diagnostic accuracy, and the Youden index [8]. P <0.05 was regarded as significant.

The diagnostic utility was calculated for: 1) single tumor marker level in the pleural fluid $(\mathrm{P})$ and serum (S); 2) single marker pleural fluid/serum level ratio (R); 3) a combination of four markers in the pleural fluid and serum. In the last case, the result was considered positive when the level of at least two markers was higher than the respective marker cut-off value.

\section{RESULTS}

There were 32 males (43\%) and $42(57 \%)$ females in the study group (mean age $65 \pm 14$ years). Unilateral pleural effusion (PE) was found in 66 patients, while 6 patients had a bilateral effusion. In 62 patients, pleural fluid met the criteria for exudates; in the remaining 12 patients pleural transudate was diagnosed. The etiologic diagnosis of 62 pleural exudates was as follows: 36 malignant PE (48.6\% of all effusions), 20 parapneumonic PE (or pleural empyema) and 6 tuberculous PE. Heart failure was responsible for the vast majority of pleural transudates ( 9 patients). In 3 patients, pleural transudate formation was related to liver cirrhosis or nephrotic syndrome. Distribution of primary tumor sites in malignant PE group was as follows: breast $(n=10)$, lung $(n=10)$, hematologic malignancies $(n=4)$, uterus $(n=3)$, malignant pleural mesothelioma $(n=2)$, stomach $(n=2)$, thyroid gland $(n=2)$, and others $(n=3)$.

All patients with non-malignant pleural exudates and patients with pleural transudates were labeled as the non-malignant PE group and compared with the patients with malignant PE. The mean age of the patients with malignant PE was $63 \pm 13$, and for the nonmalignant patients $67 \pm 14 \mathrm{yr}$. There were 10 and 20 males $(14 \%$ and $27 \%)$ in the malignant and non-malignant PE group, respectively. The comparison of median (and IQR) values of CA-125, CEA, CYFRA 21-1, and NSE concentrations in the pleural fluid (P), serum $(\mathrm{S})$, and the $\mathrm{P} / \mathrm{R}$ ratio of the respective markers in malignant and non-malignant patients are presented in Table 1.

The median pleural fluid concentrations of the investigated tumor markers were significantly higher in malignant exudates compared with non-malignant effusions, with the exception of CA-125 which was similar. An analysis of the serum concentrations revealed comparable values of CA-125 and NSE in both groups, while the CEA and CYFRA 21-1 levels were significantly higher in malignant effusions. The pleural fluid/serum concentration ratios were similar for all markers in both investigated groups, with the exception of CEA which was significantly higher in the malignant group. 
Table 1. Tumor marker concentration in pleural fluid $(\mathrm{P})$, serum $(\mathrm{S})$, and the $\mathrm{P} / \mathrm{S}$ ratio $(\mathrm{R})$ in patients with malignant vs. nonmalignant PE.

\begin{tabular}{lccc}
\hline & $\begin{array}{c}\text { Malignant effusion } \\
(\mathbf{n}=\mathbf{3 6})\end{array}$ & $\begin{array}{c}\text { Non-malignant effusion } \\
(\mathbf{n}=\mathbf{3 8})\end{array}$ & $\mathbf{P}$ \\
\hline CA-125(P) & $965.9(412.9-1790.5)$ & $518.5(297.7-796.6)$ & NS \\
CEA(P) & $12.1(1.9-79.2)$ & $1.1(0.8-1.6)$ & $<\mathbf{0 . 0 0 1}$ \\
CYFRA 21-1(P) & $27.5(14.8-213.6)$ & $12.8(5.0-31.5)$ & $<\mathbf{0 . 0 5}$ \\
NSE(P) & $2.7(0.9-10.5)$ & $1.0(0.2-4.3)$ & $<\mathbf{0 . 0 5}$ \\
CA-125(S) & $180.7(68.4-404.8)$ & $135.8(60.6-191.4)$ & NS \\
CEA(S) & $4.1(1.7-28.1)$ & $2.3(1.5-3.4)$ & $<\mathbf{0 . 0 5}$ \\
CYFRA 21-1(S) & $4.6(2.1-16.6)$ & $1.9(1.2-2.7)$ & $<\mathbf{0 . 0 0 1}$ \\
NSE(S) & $15.8(10.9-30.5)$ & $11.9(8.1-18.4)$ & NS \\
CA-125(R) & $4.6(2.6-7.1)$ & $3.7(2.6-6.9)$ & NS \\
CEA(R) & $1.4(0.8-3.9)$ & $0.5(0.4-0.7)$ & $<\mathbf{0 . 0 0 1}$ \\
CYFRA 21-1(R) & $0.1(0.06-0.8)$ & $0.1(0.02-0.3)$ & NS \\
NSE(R) & $6.9(2.1-11.1)$ & $6.9(2.6-20.5)$ & NS \\
\hline
\end{tabular}

Values are presented as median (with IQR in parenthesis). CA-125 (mU/ml), CEA $(\mu \mathrm{g} / \mathrm{ml})$, CYFRA 21-1 (ng/ml), and NSE (ng/ml).

Table 2. Receiver operating characteristics (ROC) for the four tumor markers with their cut-off points for achieving the best diagnostic accuracy.

\begin{tabular}{|c|c|c|c|c|c|c|}
\hline & Cut-off & $\begin{array}{l}\text { Sensitivity \% } \\
\quad(95 \% \mathrm{CI})\end{array}$ & $\begin{array}{l}\text { Specificity \% } \\
(95 \% \mathrm{CI})\end{array}$ & $\begin{array}{l}\text { PPV \% } \\
(95 \% \text { CI })\end{array}$ & $\begin{array}{l}\text { NPV \% } \\
(95 \% \text { CI })\end{array}$ & $\begin{array}{l}\text { Accuracy } \\
\text { AUC }\end{array}$ \\
\hline CA-125(P) & 1017 & $\begin{array}{c}50.0 \\
(32.9-67.1)\end{array}$ & $\begin{array}{c}81.6 \\
(65.7-92.4)\end{array}$ & $\begin{array}{c}72.0 \\
(50.6-87.9)\end{array}$ & $\begin{array}{c}63.3 \\
(48.3-76.6)\end{array}$ & 0.64 \\
\hline $\mathrm{CEA}(\mathrm{P})$ & 2.71 & $\begin{array}{c}72.2 \\
(54.8-85.8)\end{array}$ & $\begin{array}{c}92.1 \\
(78.6-98.3)\end{array}$ & $\begin{array}{c}89.7 \\
(72.2-97.9)\end{array}$ & $\begin{array}{c}77.8 \\
(62.9-88.8)\end{array}$ & 0.83 \\
\hline CYFRA21-1(P) & 74.7 & $\begin{array}{c}41.7 \\
(25.5-59.2)\end{array}$ & $\begin{array}{c}92.1 \\
(78.6-98.3)\end{array}$ & $\begin{array}{c}83.3 \\
(57.7-96.6)\end{array}$ & $\begin{array}{c}62.5 \\
(48.5-75.1)\end{array}$ & 0.69 \\
\hline NSE(P) & 0.22 & $\begin{array}{c}94.4 \\
(81.1-99.3)\end{array}$ & $\begin{array}{c}36.1 \\
(20.8-53.8)\end{array}$ & $\begin{array}{c}59.6 \\
(45.8-72.4)\end{array}$ & $\begin{array}{c}86.7 \\
(59.5-98.3)\end{array}$ & 0.65 \\
\hline CA-125(S) & 214.2 & $\begin{array}{c}44.4 \\
(27.9-61.9)\end{array}$ & $\begin{array}{c}78.9 \\
(62.7-90.4)\end{array}$ & $\begin{array}{c}66.7 \\
(44.7-84.4)\end{array}$ & $\begin{array}{c}60.0 \\
(45.2-73.6)\end{array}$ & 0.60 \\
\hline $\mathrm{CEA}(\mathrm{S})$ & 3.27 & $\begin{array}{c}61.1 \\
(43.5-76.9)\end{array}$ & $\begin{array}{c}73.7 \\
(54.1-84.6)\end{array}$ & $\begin{array}{c}68.7 \\
(50.0-83.9)\end{array}$ & $\begin{array}{c}66.7 \\
(50.5-80.4)\end{array}$ & 0.65 \\
\hline CYFRA 21-1(S) & 2.42 & $\begin{array}{c}72.2 \\
(54.8-85.8)\end{array}$ & $\begin{array}{c}73.7 \\
(56.9-86.6)\end{array}$ & $\begin{array}{c}72.2 \\
(54.8-85.8)\end{array}$ & $\begin{array}{c}73.7 \\
(56.9-86.6)\end{array}$ & 0.78 \\
\hline NSE(S) & 10.6 & $\begin{array}{c}80.6 \\
(64.0-91.8)\end{array}$ & $\begin{array}{c}47.4 \\
(31.0-64.2)\end{array}$ & $\begin{array}{c}59.2 \\
(44.2-73.0)\end{array}$ & $\begin{array}{c}72.0 \\
(50.6-87.9)\end{array}$ & 0.62 \\
\hline CA-125(R) & 11.16 & $\begin{array}{c}22.2 \\
(10.1-39.2)\end{array}$ & $\begin{array}{c}94.7 \\
(82.3-99.4)\end{array}$ & $\begin{array}{c}80.0 \\
(44.4-97.5)\end{array}$ & $\begin{array}{c}56.2 \\
(43.3-68.6)\end{array}$ & 0.54 \\
\hline CEA(R) & 0.83 & $\begin{array}{c}69.4 \\
(51.9-83.7)\end{array}$ & $\begin{array}{c}89.5 \\
(75.2-97.1)\end{array}$ & $\begin{array}{c}86.2 \\
(68.3-96.1)\end{array}$ & $\begin{array}{c}75.6 \\
(60.5-87.1)\end{array}$ & 0.84 \\
\hline CYFRA 21-1(R) & 11.4 & $\begin{array}{c}77.8 \\
(60.8-89.9)\end{array}$ & $\begin{array}{c}42.1 \\
(26.3-59.2)\end{array}$ & $\begin{array}{c}56.0 \\
(41.3-70)\end{array}$ & $\begin{array}{c}66.7 \\
(44.7-84.4)\end{array}$ & 0.54 \\
\hline NSE(R) & 0.04 & $\begin{array}{c}83.3 \\
(67.2-93.6)\end{array}$ & $\begin{array}{c}44.7 \\
(28.6-61.7)\end{array}$ & $\begin{array}{c}58.8 \\
(44.2-72.4)\end{array}$ & $\begin{array}{c}73.9 \\
(51.0-90.1)\end{array}$ & 0.62 \\
\hline
\end{tabular}

P - Pleural fluid values, $\mathrm{S}$ - Serum values, $\mathrm{R}$ - Ratio between pleural fluid and serum.

PPV - Positive Predictive Value; NPV - Negative Predictive Value. 


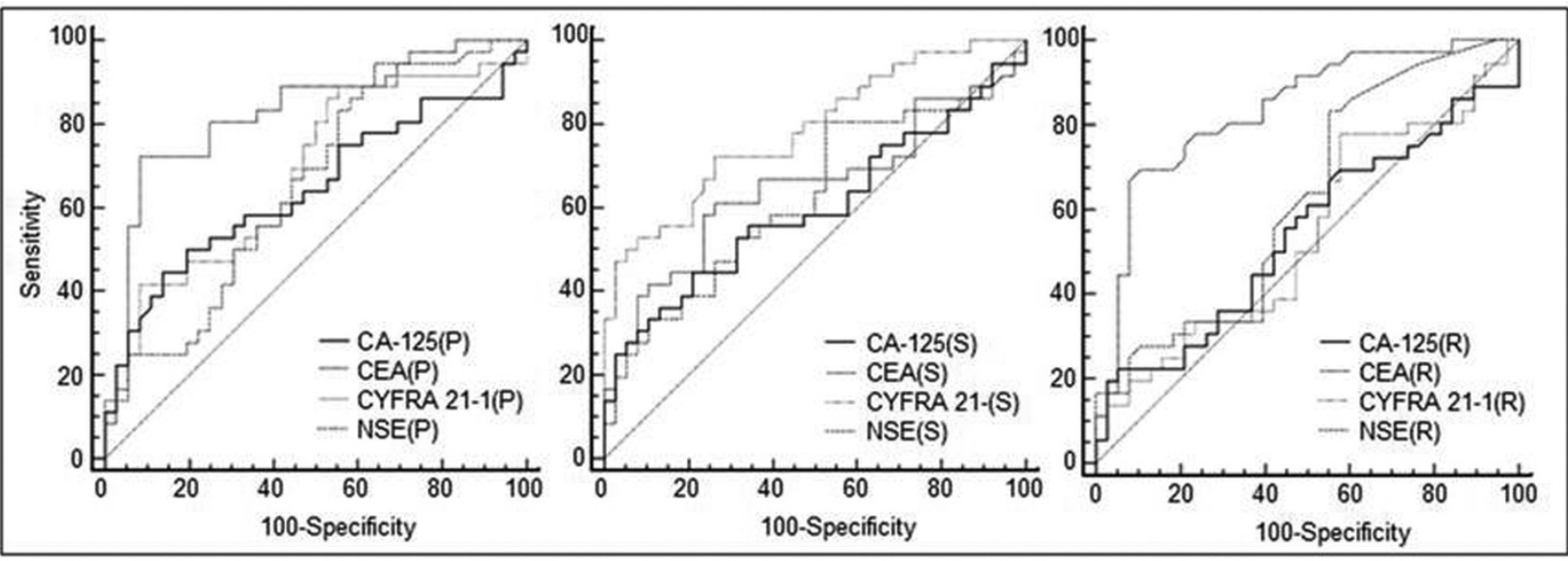

Fig. 1. Receiver operating characteristics (ROC) curves for all the investigated parameters. $\mathrm{P}$ - pleural fluid marker concentration, $\mathrm{S}$ - serum marker concentration, $\mathrm{R}-\mathrm{P} / \mathrm{S}$ ratio.
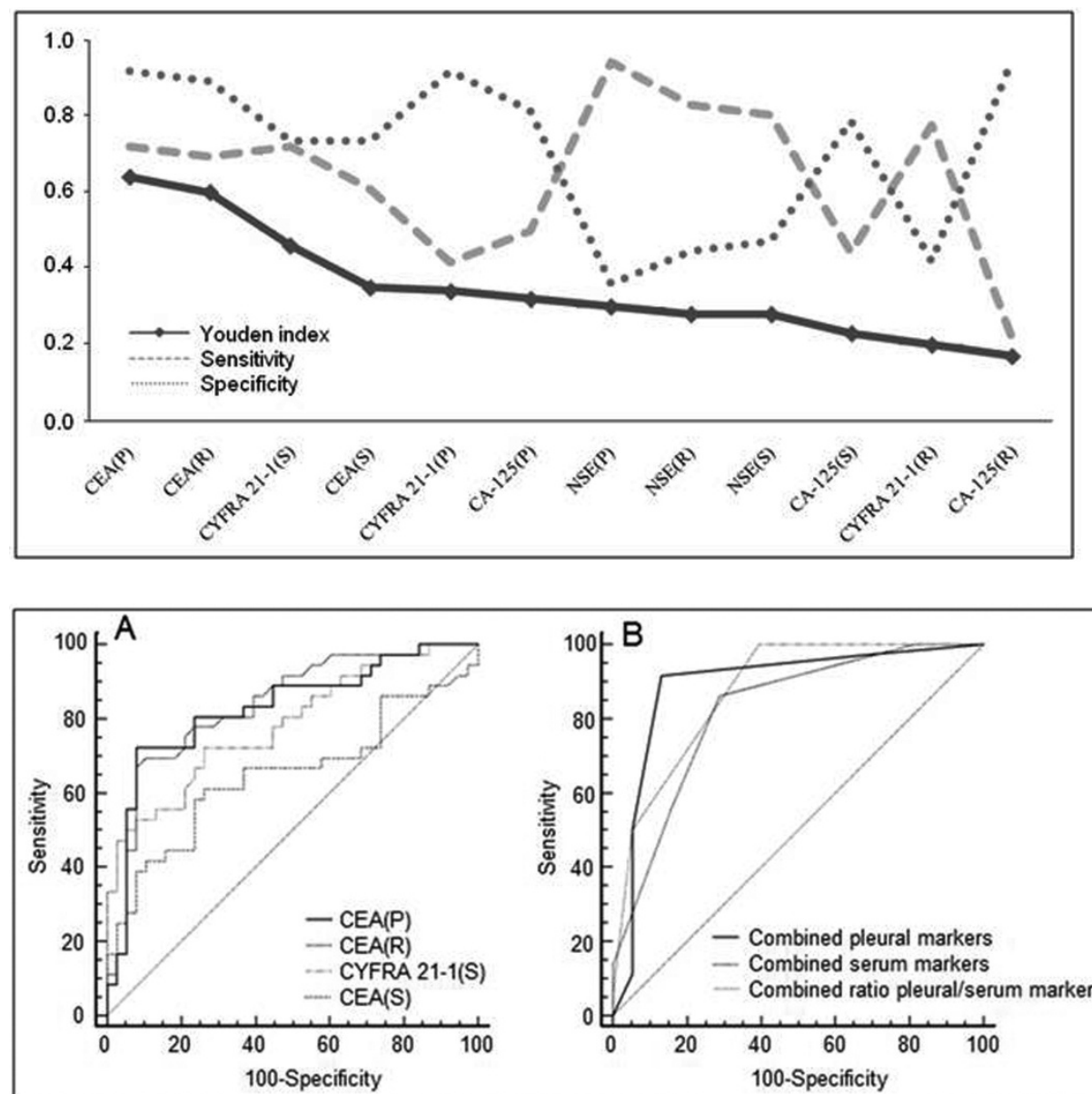

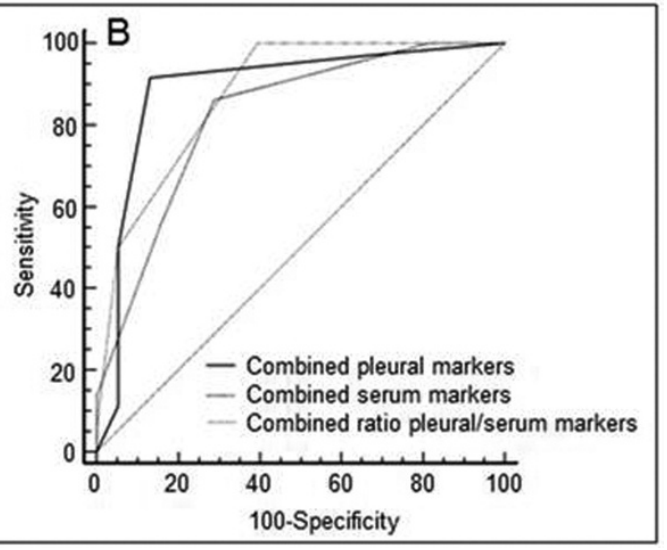

Fig. 2. Youden Index in a decreasing order, calculated from the achieved sensitivity and specificity.
The receiver operating characteristics for the four investigated tumor markers (measured in the pleural fluid $(\mathrm{P})$, serum $(\mathrm{S})$, and $\mathrm{P} / \mathrm{S}$ ratio $(\mathrm{R})$ ) with their cutoff points for achieving the best diagnostic accuracy are presented in Table 2. The ROC curves for all the investigated parameters are presented in Fig. 1.

To assess the clinical usefulness of combined sensitivity and specificity we calculated the Youden index for every investigated parameter. Fig. 2 presents the results of the Youden index in a decreasing order; the highest corresponding to higher clinical value, with its respective sensitivity and specificity. The four highest values calculated with the help of the Youden index are presented on a ROC curve. The highest clinical value was demonstrated for the CEA pleural and serum concentrations, along with the $\mathrm{P} / \mathrm{S}$ ratio (Fig. $3 \mathrm{~A})$.

A combination of the ROC curves for all investigated parameters (pleural fluid and serum tumor marker concentrations with the $\mathrm{P} / \mathrm{S}$ ratio) resulted in a higher sensitivity and specificity. When at least two pleural fluid marker levels were above their cut-off points, the sensitivity and specificity achieved $91.7 \%$ and $86.6 \%$, respectively. An analogous analysis for the serum markers revealed the sensitivity and specificity of $86.1 \%$ and $71.1 \%$, respectively. ROC analysis for 
combined tumor marker $\mathrm{P} / \mathrm{S}$ ratio showed a very high sensitivity $(100 \%)$, but relatively low specificity $(60.5 \%)$ (Fig. 3B). The Youden indices for the three above mentioned combinations were $0.76,0.57$ and 0.61 , respectively. The Youden index achieved the highest value for the combined four pleural fluid marker measurements compared with those for each single marker; higher than those for the combined serum markers or combined ratio of pleural/serum markers.

\section{DisSCUSSION}

Thoracentesis is a relatively simple and safe procedure which allows obtaining pleural fluid samples in patients with PE. One of the most important components of the routine PE diagnostic work-up is its cytological examination. The mean sensitivity of the cytological examination in subjects with malignant PE is estimated at $60 \%$. This means that in about $40 \%$ of malignant PE, the fluid cytology gives a false negative result and does not confirm the malignant origin of the effusion. Thus, to determine the PE etiology, more invasive procedures like closed pleural biopsy or medical thoracoscopy are required. Understandably, attempts have focused on various tumor markers in pleural fluid and serum. However, no single tumor marker with a satisfactory sensitivity and specificity has yet been identified. To increase the diagnostic accuracy of the tumor marker measurements, combinations of selected markers have been studied. These combinations included markers assumed to guarantee the highest probability in the detection of malignancy of different origin and type.

In the present study we chose tumor markers used for detection and monitoring of non-small cell lung carcinoma and mesothelioma (CYFRA 21-1), small cell lung carcinoma (NSE), ovarian (CA-125), and digestive and breast cancers (CEA), trying to cover the main etiologic spectrum of malignant PE. CEA in PE proved to be the marker with the highest diagnostic accuracy. The area under curve (AUC) in the ROC analysis for this parameter was 0.83 . Previous studies which included this marker revealed the following AUC values: $0.97 ; 0.84$, and $0.72[3,9,10]$. The diagnostic accuracy for serum measurements in our study was lower and equaled to 0.65 . In a study by Lee and Chang [10], the AUC for serum CEA concentrations was 0.97 , while in that by Romaro et al [9] it was 0.75 . The discrepancy may be due to differences in the etiology of the analyzed pleural fluids. The highest diagnostic accuracy was found for patients with lung cancer. CEA is a glycoprotein component of the glycocalyx of the endodermic epithelium, which is abundant in a wide variety of tumors. It has been suggested that increased pleural fluid CEA level may be caused by direct or indirect mechanisms (pleural invasion or decreased lymphatic drainage due to lymphatic obstruction $[2,11]$. In the present study, the diagnostic accuracy for the pleural fluid/serum CEA level ratio was 0.84; therefore, it seems that serum CEA measurements do not add relevant diagnostic information.

CYFRA 21-1 is another marker with a high diagnostic yield, but contrary to the three other markers, the serum, rather than plural fluid, level of CYFRA 21-1 shows a higher overall diagnostic accuracy. CYFRA 21-1, soluble fragments of cytokeratin 19, is expressed by all histological types of lung cancer, especially by squamous lung cancer [12]. Similarly to CEA, increased levels of CYFRA 21-1 in the pleural fluid may result from impaired lymphatic drainage or cancer involvement of the pleura. In our study, the AUC for this marker in serum was 0.78 . In a series studied by Li et al [13], CYFRA 21-1 serum concentration distinguished malignant from non-malignant effusions with $56.3 \%$ sensitivity, $86.7 \%$ specificity, and a diagnostic accuracy of 0.71 . In another analysis of lung cancer-associated PE, the accuracy was higher and equaled to 0.83 [10]. AUC for pleural fluid concentrations of this marker was 0.71 and was comparable to the 0.69 achieved in the present study. It seems, therefore, that CYFRA 21-1 is a valuable marker in the diagnosis of PE related to lung cancer. In our series of the 36 patients with malignant PE, lung cancer was diagnosed only in $6(16.7 \%)$. This may explain why the diagnostic accuracy of CYFRA 21-1 pleural measurements in the present study was relatively low $-41.7 \%$. Studies in which lung cancer was the predominant cause of PE, revealed a higher diagnostic yield of this parameter. Serum CYFRA 21-1 has been found useful for the diagnosis of lung cancer, particularly of the squamous cell type. However, Dejsomritrutai et al [14] found that CYFRA 21-1 is a good marker also in a population of patients with PE caused by adenocarcinoma. These authors reported CYFRA 21-1 sensitivity of $81.5 \%$ and $74.1 \%$ in serum and the pleural fluid, respectively. The specificity of this marker was also high and reached as much as $97.1 \%$ for both serum and PE.

We found a high sensitivity, but low diagnostic accuracy, for NSE. NSE is a known marker of small cell carcinoma. The sensitivity of pleural fluid NSE concentration was as high as $94.4 \%$, while for the serum measurements it was $80.6 \%$. However, the specificity of this marker was relatively low, thus the AUC reached 0.65 for pleural fluid and 0.62 for serum. A similar diagnostic accuracy was observed in previous reports (0.57 and 0.58, respectively) [10]. In the current study, pleural fluid NSE was significantly higher in malignant than benign effusions. The heterogeneity of the investigated subjects does not allow singling out small cell lung cancer related exudates from all PE.

CA-125 is particularly useful in detecting the recurrence of ovarian cancer [15], although the marker is also observed in malignancies of the endometrium, lung, breast, and gastrointestinal tract. In our patients, the accuracy of CA-125 measurement in PE and serum was 0.64 and 0.60 , respectively. Other authors report a $37 \%$ sensitivity and $100 \%$ specificity at a cutoff value of $1000 \mathrm{U} / 1$ [2]. To compare this data, we calculated the Youden index. In a study by Ferrer et al [2], the Youden index reached 0.37, while in the present study the respective value was 0.32 , which, we believe, is comparable.

Combining the markers may improve the diagnostic accuracy. Ferrer et al [2] achieved 65\% sensitivity and $100 \%$ specificity for a combination of CYFRA 21-1, CEA, and CA-125 assessed in pleural fluid. The sensi- 
tivity of these combined measurements was higher than that of cytological examination (56\%) [2]. In the present study, a combination of four markers (CEA, CA-125, CYFRA 21-1, and NSE), with a cut-off for two or more markers, gave sensitivity and specificity of $91.7 \%$ and $86.8 \%$ for pleural measurements, $86.1 \%$ and $71.1 \%$ for serum, and $100 \%$ and $60.5 \%$ for the pleural fluid/ serum ratio, respectively. The AUC calculated from the ROC analysis achieved as much as $0.89,0.82$, and 0.88 for the pleural fluid, serum, and pleural fluid/serum ratio, respectively. It seems that the pleural fluid/serum marker ratio does not add any essential clinical information. A meta-analysis by Liang et al [16] showed similar results with the combinations of different tumor markers, e.g., the AUC for CEA/CA-125 was 0.86 and for CEA/ CYFRA 21-1 0.93 .

As it could be expected, the diagnostic yields of different tumor markers evaluated with the Youden index and from the area under the curve in the ROC analysis were similar. The Youden index is a useful parameter enabling a comparative analysis of data found by various authors when single values of sensitivity and specificity are reported. This statistical method was successfully used in the meta-analysis of the usefulness of adenosine deaminase and interferon gamma measurements for the diagnosis of tuberculous pleurisy [17]. In the present study, the diagnostic yield of the three most accurate markers $\mathrm{CEA}(\mathrm{P}), \mathrm{CEA}(\mathrm{R})$, CYFRA 21-1(P) calculated with the Youden index was virtually the same as assessed with the AUC.

The present study had some limitations. There were relatively small numbers of patients in malignant and non-malignant PE groups. Also, as many as 28 patients were withdrawn from the study because the etiology of the effusion remained unknown. Finally, variability of diseases responsible for PE was rather limited (malignant, parapneumonic, empyemic, and tuberculous exudates). Nevertheless, we believe we have shown that there are significant differences between the diagnostic value of various $\mathrm{PE}$ and serum markers. Overall, pleural fluid markers are superior to serum markers in determining the pleural fluid etiology. A combination of two or more tumor markers may help improve their diagnostic accuracy. However, it seems that measurements of pleural fluid and serum tumor markers play a limited role in the differentiation between malignant and non-malignant PE.

Conflicts of interest: No conflicts of interest were declared in relation to this work.

\section{REFERENCES}

[1] Loddenkemper R. Thoracoscopy - state of the art. Eur Respir J 1998; 11: 213-21.

[2] Ferrer J, Villarino MA, Encabo G, Felip E, Bermejo B, Vilà S, Orriols R. Diagnostic utility of CYFRA 21-1, carcinoembryonic antigen, CA 125, neuron specific enolase, and squamous cell antigen level determinations in the serum and pleural fluid of patients with pleural effusions. Cancer 1999; 86: 1488-95.
[3] Porcel JM, Vives M, Esquerda A, Salud A, Pérez B, Rodríguez-Panadero F. Use of a panel of tumor markers (carcinoembryonic antigen, cancer antigen 125, carbohydrate antigen 15-3, and cytokeratin 19 fragments) in pleural fluid for the differential diagnosis of benign and malignant effusions. Chest 2004; 126: 1757-63.

[4] Krenke R, Safianowska A, Paplinska M, Nasilowski J, Dmowska-Sobstyl B, Bogacka-Zatorska E, Jaworski A, Chazan R. Pleural fluid adenosine deaminase and interferon gamma as diagnostic tools in tuberculosis pleurisy. J Physiol Pharmacol 2008; 59 Suppl 6: 349-60.

[5] Sahn SA. Pleural diseases related to metastatic malignancies. Eur Respir J 1997; 10: 1907-13.

[6] Valdés L, Pose A, San José E, Martinez Vázquez JM. Tuberculous pleural effusions. Eur J Intern Med 2003; 14: 77-88.

[7] Hamm H, Light RW. Parapneumonic effusion and empyema. Eur Respir J 1997; 10: 1150-6.

[8] Youden WJ. Index for rating diagnostic tests. Cancer 1950; 3: $32-5$.

[9] Romero S, Fernández C, Arriero JM, Espasa A, Candela A, Martín C, Sánchez-Payá J. CEA, CA 15-3 and CYFRA 21-1 in serum and pleural fluid of patients with pleural effusions. Eur Respir J 1996; 9: 17-23.

[10] Lee JH, Chang JH. Diagnostic utility of serum and pleural fluid carcinoembryonic antigen, neuron-specific enolase, and cytokeratin 19 fragments in patients with effusions from primary lung cancer. Chest 2005; 128: 2298303.

[11] Gu P, Huang G, Chen Y, Zhu C, Yuan J, Sheng S. Diagnostic utility of pleural fluid carcinoembryonic antigen and CYFRA 21-1 in patients with pleural effusion: a systematic review and meta-analysis. J Clin Lab Anal 2007; 21: 398-405.

[12] Lai RS, Chen CC, Lee PC, Lu JY. Evaluation of cytokeratin 19 fragment (CYFRA 21-1) as a tumor marker in malignant pleural effusion. Jpn J Clin Oncol 1999; 29: 421-4.

[13] Li CS, Cheng BC, Ge W, Gao JF. Clinical value of CYFRA21-1, NSE, CA15-3, CA19-9 and CA125 assay in the elderly patients with pleural effusions. Int J Clin Pract 2007; 61: 444-8.

[14] Dejsomritrutai W, Senawong S, Promkiamon B. Diagnostic utility of CYFRA 21-1 in malignant pleural effusion. Respirology 2001; 6: 213-6.

[15] Osman N, O'Leary N, Mulcahy E, Barrett N, Wallis F, Hickey K, Gupta R. Correlation of serum CA125 with stage, grade and survival of patients with epithelial ovarian cancer at a single centre. Ir Med J 2008; 101: 245-7.

[16] Liang QL, Shi HZ, Qin XJ, Liang XD, Jiang J, Yang HB. Diagnostic accuracy of tumour markers for malignant pleural effusion: a meta-analysis. Thorax 2008; 63: 35-41.

[17] Greco S, Girardi E, Masciangelo R, Capoccetta GB, Saltini $C$. Adenosine deaminase and interferon gamma measurements for the diagnosis of tuberculous pleurisy: a meta-analysis. Int J Tuberc Lung Dis 2003; 7: 777-86.

\section{Corresponding author:}

P. Korczynski

Department of Internal Medicine, Pneumology and

Allergology

Medical University of Warsaw

Banacha 1A St.

02-097 Warsaw

Poland

Phone: +48225992753

Fax: $\quad+48225991560$

E-mail: Piotr.korczynski@wum.edu.pl 